Madera y Bosques vol. 25, núm. 2, e252550 Verano 2019 Artículos científicos

\title{
Tratamientos de preservación de Bambusa vulgaris vittata contra el ataque de Dinoderus minutus
}

\section{Preservation treatments of Bambusa vulgaris vittata against the attack of Dinoderus minutus}

\author{
Juliana Cortez-Barbosa',2, Elen Aparecida Martines Morales',2, Karina Arakaki Higuti', Ana Clara Lancarovici Alves', \\ Victor Almeida De Araujo ${ }^{1,3,},{ }^{*}$ Maristela Gava ${ }^{1,2}$ y José Nivaldo Garcia ${ }^{3}$
} Grupo de Investigación en Desarrollo de los 2 Universidad del Estado de São Paulo. Curso de
Productos Lignocelulósicos (LIGNO), Itapeva, Brasil.
$\begin{aligned} & \text { Ingeniería Industrial de la Madera. Campus de } \\ & \text { Itapeva. Itapeva, Brasil. }\end{aligned}$

\author{
3 Universidad de São Paulo. Departamento de \\ Ciencias Forestales. Escuela de Agricultura Luiz de \\ Queiroz. Piracicaba, Brasil. \\ * Autor de correspondencia. engim.victor@yahoo.de
}

\section{RESUMEN}

Las cañas de bambú son un material de construcción único para diversos tipos de estructuras y cada vez hay una mayor aceptación de los bambúes para usos estructurales. Sin embargo, tienen como inconveniente su propensión al ataque por insectos. En este trabajo se hizo un estudio de la eficacia de tres diferentes tipos de preservadores: 1) químicos tradicionales, 2) naturales de bajo riesgo para el ser humano y 3) térmicos a diferentes temperaturas, para prevenir el ataque del insecto escarabajo Dinoderus minutus al bambú de la especie Bambusa vulgaris vittata. Los dos primeros tratamientos se realizaron por inmersión y el tipo térmico fue hecho por el proceso de secado. Cada dos semanas, se evaluó el ataque a tres especímenes de cada tratamiento, incluyendo los correspondientes a un testigo sin elemento preservador alguno. Los resultados mostraron que la mayoría de los tratamientos por inmersión fueron eficaces, reduciendo considerablemente el ataque de los insectos en comparación con el testigo. En cuanto al tratamiento térmico, las temperaturas menores o iguales a $130{ }^{\circ} \mathrm{C}$ no mostraron ser eficaces; las temperaturas de $190{ }^{\circ} \mathrm{C}$ y $210{ }^{\circ} \mathrm{C}$ revelaron eficacias aceptables.

PALABRAS CLAVE: bambú, gorgojo del bambú, preservación de bambú, preservadores, tratamiento térmico, tratamiento de inmersión.

\section{ABSTRACT}

Bamboo canes are a unique construction raw material for several types of structures, and there is a growing acceptance of bamboos for structural uses. However, they have as a disadvantage their propensity to attack by insects. In this work, a study of the effectiveness of three different types of preservatives was made with: 1) traditional chemicals, 2) natural substances with low risk to humans and 3) thermal at different temperatures to prevent the Dinoderus minutus beetle attack to Bambusa vulgaris vittata bamboo species. The first two treatments were performed by immersion, and thermal type was made by a drying process. Every two weeks, the attack on three specimens of each treatment was evaluated, including those corresponding to a control specimen without any preservation element. The results showed that most of the treatments by immersion were effective, reducing considerably the attack of the insects in comparison with the control. As for the heat treatment, temperatures less than or equal to $130^{\circ} \mathrm{C}$ did not prove to be effective; temperatures of 190 ${ }^{\circ} \mathrm{C}$ and $210{ }^{\circ} \mathrm{C}$ revealed acceptable efficiencies.

KEYWORDS: bamboo, bamboo weevil, bamboo preservation, preservatives, thermal treatment, immersion treatment. 


\section{INTRODUCCIÓN}

El bambú es similar a un árbol en cuanto a que tiene una parte aérea (culmos, hojas y ramas) y otra subterránea (rizomas y raíces), adicionalmente, su estructura celular tiene un tejido y propiedades similares a la madera (Dunkelberg, 1996). Los bambúes están ganando cada vez más atención como un cultivo alternativo de múltiples usos y beneficios. Estas hierbas leñosas de larga duración son generalmente perennes en los climas a los que están adaptados (Diver, 2001).

El bambú juega un papel importante contra la erosión, porque se puede sembrar en terrenos difíciles e incluso utilizarse en las zonas degradadas donde otras plantas no podrían desarrollarse. Otra ventaja de su uso es que el bambú es una fuente renovable de rápido crecimiento, en comparación con la madera. Ordóñez-Candelaria (1999) señala que su rendimiento por hectárea es similar o mayor que de muchas especies maderables. El bambú es un material renovable y de bajo costo, que se encuentra en abundancia en las regiones tropicales y subtropicales (Espelho y Beraldo, 2008).

El potencial del bambú ha sido poco utilizado en varios países latinoamericanos, por ejemplo, Brasil (Arruda, del Menezzi, Teixeira y de Araújo, 2011). En general, para mejorar el sector del bambú y asegurar su competitividad como una fuente alternativa a la madera, se requiere una política pública inclusiva para poner en práctica las intervenciones pertinentes (Obiri y Oteng-Amoako, 2007).

El culmo es la parte del bambú más utilizada en la industria forestal (construcción, muebles, artesanías, entre otros), sin embargo, la información tecnológica de la especie está poco documentada (Zaragoza-Hernández, Borja de la Rosa, Zamudio-Sánchez, Ordóñez-Candelaria y Bárcenas-Pazos, 2014). Las cañas de bambú son un material de construcción único para los diversos tipos de estructuras y cada vez tiene una mayor aceptación para usos estructurales; sin embargo su uso es limitado por su propensión a la degradación biológica (Liese y Tang, 2015). La preservación del bambú para aumentar su vida útil es restringida debido a razones económicas (Liese, 1994).
Es indudable que los culmos del bambú tienen una gran variación en sus dimensiones a lo largo del mismo, además de que su sección transversal dista mucho de ser un círculo perfecto (Ordóñez-Candelaria y Salomón Quintana, 2009), sin embargo, pueden ser eficientemente aplicados en la construcción civil. Además del uso en la construcción de viviendas, el bambú también se utiliza en la fabricación de escaleras de mano, muebles, defensas flotantes, artesanía, cestas, etc. (Kaur, Pant, Satya y Naik, 2014; Kurhekar, Moola, Shinde y Kolhe, 2015a; Kurhekar, Sanap y Kolhe, 2015b).

El bambú ha sido señalado como una alternativa adecuada para ser utilizada en la construcción de viviendas sociales, debido a su potencial para reducir los costos, ofreciendo el mismo nivel de calidad de las tecnologías convencionales (Barboza, Barbirato y Silva, 2008). Un ambiente sostenible sería posible más rápido con la implementación de la producción industrial de bambú que con la de madera (Escamilla, Habert y Wohlmuth, 2016). El bambú es uno de los materiales estructurales más fuertes de base biológica, pero sucumbe al daño físico y mecánico por algunos factores abióticos y es susceptible a la degradación por organismos similares a los que atacan a la madera (Liese y Tang, 2015).

Microscópicamente, el bambú comprende vasos conductores, fibras y células de parénquima (Vasconcellos, 2004). Contiene también otra composición orgánica además de la celulosa y la lignina, por ejemplo, puede contener almidón $(2 \%-6 \%)$, sacáridos desoxidados $(2 \%)$, grasa $(2 \%-4 \%)$ y proteína $(0,8 \%-6 \%)$ (Liese, 1980). El almidón en los culmos es la principal característica química del bambú para el uso industrial como materia-prima y energía (Azzini y Beraldo, 2000). Ese almidón es una característica atractiva para los agentes de deterioro (Nunes, 2005; Liese y Tang, 2015), especialmente los de perforación como Dinoderus minutus (Fig. 1); lo anterior constituye su mayor debilidad (Nunes, 2005). El gorgojo del bambú (Dinoderus minutus) se ha encontrado en chips de yuca, ñame seco, bambú, maíz almacenado y corteza de árboles (Schäfer, Goergen y Borgemeister, 2000). Problemático en 


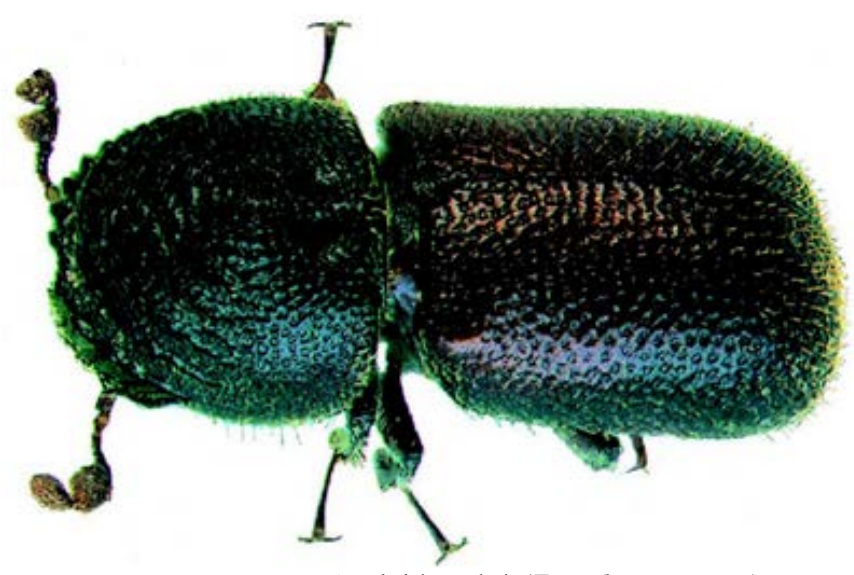

FIGURA 1. Insecto gorgojo del bambú (Dinoderus minutus). Fuente: Schäfer et al. (2000).

zonas de almacenamiento de bambú, el gorgojo puede ser tan abundante que llega a causar graves daños, reduciendo el bambú a polvo o fibras, eso si no lo destruye por completo (Singh y Bhandari, 1988).

$\mathrm{El}$ ataque de este insecto al bambú es un problema para fines estructurales y para la fabricación de muebles, pues para estos fines son importantes sus propiedades mecánicas y su estética final.

De acuerdo con Nogueira (2009), para asegurar la vida útil de las piezas de bambú, es necesario tratarlas. El tratamiento contra ataque de insectos es un requisito importante para la utilización de este recurso lignocelulósico valioso (Liese y Tang, 2015). La función de los preservadores químicos es, básicamente, inhibir el desarrollo y la vida de los microorganismos, sin afectar los tejidos, ni cambiar el color natural del material. Para esto, existen muchos tratamientos preservadores, el más adecuado depende del uso final que se dará al bambú. Los tratamientos existentes son de productos químicos y pueden ser muy tóxicos para el ser humano y no aptos para determinados fines; por ejemplo, cuando el uso final del bambú está vinculado con los alimentos. Los tratamientos más naturales que no perjudiquen la salud pueden ser considerados ineficaces porque no presentan la alta eficiencia que los químicos, tal vez por el método de impregnación, o incluso por la eficacia del principio activo de conservadores y fijadores.
Para el tratamiento de bambúes, la literatura describe el uso de varios preservadores químicos, por ejemplo, pentaclorofenol (Galvão, 1967) y borato de cobre cromatado (Espelho y Beraldo, 2008; Tiburtino, Paes, Beraldo, Arantes y Brocco, 2015) entre otros. Sin embargo, estas sustancias son demasiado tóxicas. Por eso, es muy importante la búsqueda de nuevos preservadores químicos menos agresivos tanto para el ser humano como para el medio ambiente. Un buen ejemplo puede ser el estudio de Kaur et al. (2014), que se refiere a la utilización de extractos $\mathrm{y}$ aceites naturales.

Los modos de impregnación también son numerosos, pero el método de inmersión es el más económico, porque solo exige un recipiente para poner el preservador; mientras que el costo del tratamiento en autoclave es mayor.

Al mismo tiempo, el secado de los bambúes puede ser otra alternativa viable. De acuerdo con Liese y Tang (2015), es necesario el secado de bambú antes de su uso, ya que cuando está seco es más fuerte y menos susceptible al ataque de insectos que cuando está húmedo. También la contracción y el hinchamiento del bambú están relacionados con su contenido de humedad.

Meng, Yu, Zhang, Yu y Gao. (2016) comprobaron que los tratamientos térmicos de los bambúes propician que el contenido de hemicelulosa y celulosa disminuyan, mientras que el contenido de lignina se incrementa, es decir, hacen que su estabilidad dimensional y durabilidad mejoren.

En este trabajo se analizó la eficacia de tres diferentes tipos de preservadores: 1) químicos tradicionales (pentox y ácido bórico), 2) naturales de bajo riesgo para el ser humano (extracto de Melia azedarach, extracto de Azadirachta indica, ácido piroleñoso de Eucalyptus sp y ácido piroleñoso de Bambusa vulgaris vittata) y 3) térmicos a diferentes temperaturas $\left(50^{\circ} \mathrm{C}, 70^{\circ} \mathrm{C}, 90^{\circ} \mathrm{C}, 110^{\circ} \mathrm{C}, 130^{\circ} \mathrm{C}, 150{ }^{\circ} \mathrm{C}\right.$, $170^{\circ} \mathrm{C}, 190^{\circ} \mathrm{C}$ y $210^{\circ} \mathrm{C}$ ), para prevenir los posibles ataques de los escarabajos Dinoderus minutus al bambú Bambusa vulgaris. La idea de utilizar estos tratamientos, con la excepción de pentox, fue buscar nuevas alternativas a los tratamientos que no dañen el medio ambiente y sean 
biodegradables; esto en el entendido de que hasta este momento los productos químicos son el principal medio de protección. Se decidió probar el tratamiento con pentox para comparar los resultados obtenidos, pues se trata de un producto químico ligeramente nocivo para el ambiente, pero con probada eficacia contra el ataque de insectos a la madera. La elección del ácido bórico se debió a su amplio uso comercial. Los otros tratamientos (ácidos piroleñosos de eucalipto y de bambú y los extractos de neem y de cinamomo) fueron seleccionados por su carácter menos contaminante y riesgoso a los seres humanos.

\section{OBJETIVOS}

Analizar el efecto preservador frente a los insectos de dos tratamientos químicos tradicionales (ácido bórico y pentox), cuatro tratamientos naturales de bajo riesgo para el ser humano (ácidos piroleñosos de eucalipto y de bambú y extractos de neem y de cinamomo) y uno térmico a nueve diferentes temperaturas (entre $50^{\circ} \mathrm{C}$ y $210^{\circ} \mathrm{C}$ ), aplicados a la especie Bambusa vulgaris vittata contra los ataques de los gorgojos del bambú (Dinoderus minutus).

\section{MATERIALES Y MÉTODOS}

Debido a la falta de documentos normativos para realizar pruebas específicas en bambú (Fig. 2), se adaptó la norma EN-310/2000 (European Committee for Standardization, 2000) para la definición de los especímenes. Las dimensiones quedaron de la siguiente manera: $50 \mathrm{~mm}$ de anchura, espesor original del culmo y longitud dependiente del espesor, 20 veces el espesor original más $50 \mathrm{~mm}$. Por la misma carencia de documentos técnicos específicos, la medición de las eficiencias de cada tratamiento se ha realizado por medio de la cantidad de perforaciones en los especímenes evaluados.

Para cada tratamiento se probaron tres especímenes, obtenidos de culmos con más de 3 años de edad, provenientes del Estado de São Paulo, Brasil. Entre los tratamientos por inmersión probados, se encuentran los dos químicos (pentox con concentración de $2 \%$ y ácido bórico con concentración de 6\%) y los cuatro naturales de bajo riesgo para el ser humano [extracto de cinamomo (Melia azedarach), extracto de neem (Azadirachta indica), ácido piroleñoso de madera (Eucalyptus sp) y ácido piroleñoso de bambú (Bambusa vulgaris vittata) - cuyas concentraciones fueron de $10 \%$ para cada uno]. El tratamiento térmico se realizó a nueve temperaturas: $50^{\circ} \mathrm{C}, 70^{\circ} \mathrm{C}, 90^{\circ} \mathrm{C}, 110{ }^{\circ} \mathrm{C}$, $130{ }^{\circ} \mathrm{C}, 150{ }^{\circ} \mathrm{C}, 170{ }^{\circ} \mathrm{C}, 190{ }^{\circ} \mathrm{C}$ y $210{ }^{\circ} \mathrm{C}$. Para lograr una buena impregnación, la inmersión de cada tratamiento duró dos días, excepto para el pentox. En este tratamiento, los especímenes se sumergieron durante 1 minuto, siguiendo las directrices especificadas en el propio producto.

Con el tratamiento térmico, en total se probaron 27 especímenes, tres para cada intervalo de temperatura. El tratamiento se inició colocando en la estufa, con temperatura ambiente, todos los especímenes que se someterían a todas las temperaturas.

Se inició el incremento de temperatura y, al alcanzar la temperatura deseada, los especímenes de cada temperatura en orden ascendente se fueron transfiriendo a otra estufa, de modo que pudieran mantener esa temperatura durante 1 hora y luego el horno se apagó a la temperatura ambiente gradualmente. Los intervalos de temperatura por tratamiento pueden entenderse mejor a través de la figura 3. Este proceso se basa en experimentos de Brito, Garcia, Bortoletto Junior, Pessoa y Silva (2006), quienes trataron térmicamente ejemplares de madera, y de Colla, Beraldo y Brito (2011), quienes también estudiaron este tipo de tratamiento con bambú.

Brito et al. (2006) levantaron la temperatura a una velocidad de aproximadamente $0,14^{\circ} \mathrm{C}$ por minuto. En este estudio, con el fin de concluir el tratamiento térmico en un día, la tasa de incremento en temperatura fue de $6{ }^{\circ} \mathrm{C}$ a cada 30 minutos.

Después del tiempo especificado para cada tipo de tratamiento, los especímenes se colocaron en cajones abiertos y aireados, favoreciendo los simulacros de ataque (Fig. 4). 

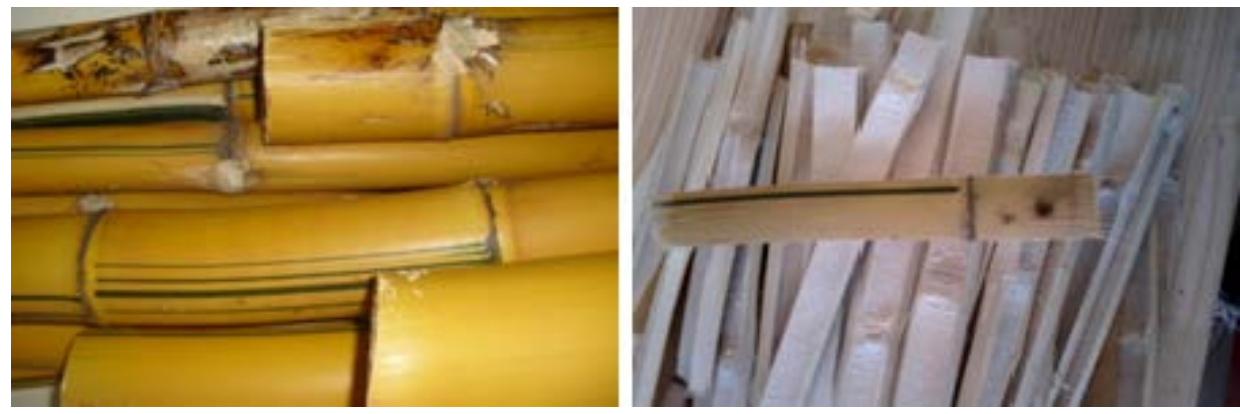

FIGURA 2. Bambúes Bambusa vulgaris vittata y muestras sin tratamiento y sin ataque.

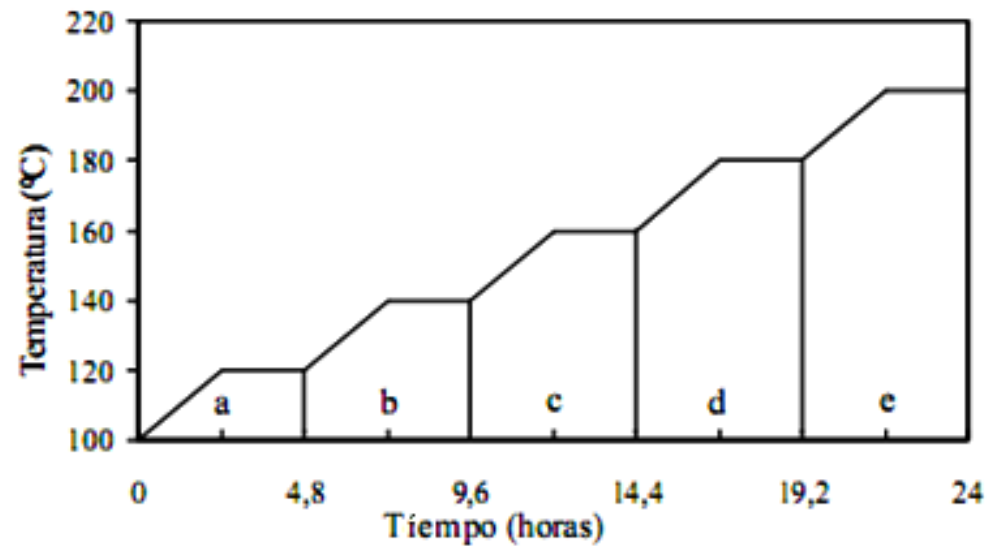

FIGURA 3. Condición de temperatura y tiempo en el tratamiento térmico
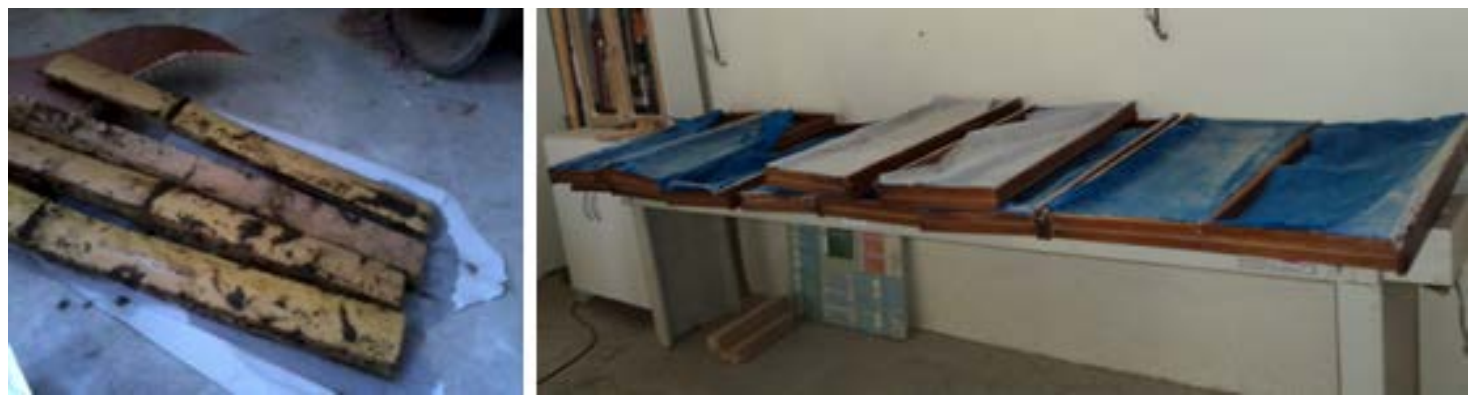

FIGURA 4. Especímenes tratados con neem en el proceso de secado (izquierda) y almacenamiento local de las muestras (derecha).

Quincenalmente, se verificó el número de ataques sufridos por cada espécimen. Cada nuevo ataque fue marcado para facilitar el recuento. Se registró el número de ataques por probeta para cada tratamiento, se calculó la media de los ataques por tratamiento y su desviación estándar.

\section{Resultados}

Los resultados de ataques de Dinoderus minutus a los especímenes de Bambusa vulgaris vittata sometidos a los distintos tratamientos se muestran en la tabla 1. La figura 5 muestra los ataques de insectos sufridos por los especímenes sometidos a tratamientos de inmersión y en la figura 6 se aprecian los ataques sufridos por los especímenes sometidos a tratamiento por temperatura.

Como se puede observar, la mayoría de los ataques sufridos están cerca de los nodos. Esto sucede debido a un mayor contenido de almidón presente en ese lugar, si se compara con otras zonas del bambú. No hubo ningún ataque en la región exterior, que es donde se concentra una mayor cantidad de fibra y menos almidón. 
TABLA 1. Detalle del número de ataques de Dinoderus minutus a Bambusa vulgaris vittata, por espécimen y valor medio.

\begin{tabular}{lccccc}
\hline Tratamientos & $P 1$ & $P 2$ & $P 3$ & media P & $D E$ \\
\hline Control & 28 & 33 & 50 & 37,0 & 21,36 \\
Extracto de CNM & 1 & 12 & 2 & 5,0 & 2,89 \\
ACP & 0 & 1 & 10 & 3,7 & 2,12 \\
ACBV & 0 & 4 & 0 & 1,3 & 0,77 \\
AB & 0 & 0 & 0 & 0,0 & 0,00 \\
Pentox & 0 & 0 & 0 & 0,0 & 0,00 \\
Extracto de NM & 17 & 0 & 6 & 7,7 & 4,43 \\
Tratamiento térmico a $50^{\circ} \mathrm{C}$ & 16 & 29 & 15 & 20,0 & 11,55 \\
Tratamiento térmico a $70^{\circ} \mathrm{C}$ & 5 & 6 & 4 & 5,0 & 2,89 \\
Tratamiento térmico a $90^{\circ} \mathrm{C}$ & 16 & 17 & 6 & 13,0 & 7,51 \\
Tratamiento térmico a $110^{\circ} \mathrm{C}$ & 0 & 4 & 10 & 4,7 & 2,69 \\
Tratamiento térmico a $130^{\circ} \mathrm{C}$ & 24 & 13 & 20 & 19,0 & 10,97 \\
Tratamiento térmico a $150^{\circ} \mathrm{C}$ & 6 & 0 & 0 & 2,0 & 1,15 \\
Tratamiento térmico a $170^{\circ} \mathrm{C}$ & 8 & 7 & 2 & 5,7 & 3,27 \\
Tratamiento térmico a $190^{\circ} \mathrm{C}$ & 0 & 2 & 0 & 0,7 & 0,38 \\
Tratamiento térmico a $210^{\circ} \mathrm{C}$ & 0 & 0 & 0 & 0,0 & 0,00 \\
\hline
\end{tabular}

CNM: cinamomo; NM: neem; ACP: acido piroleñoso de eucalipto; ACBV: ácido piroleñoso de bambú; AB: ácido bórico; P1, P2 y P3: ataques en especímenes 1, 2 y 3; Media P: media de los ataques en los especímenes; DE: desviación estándar.
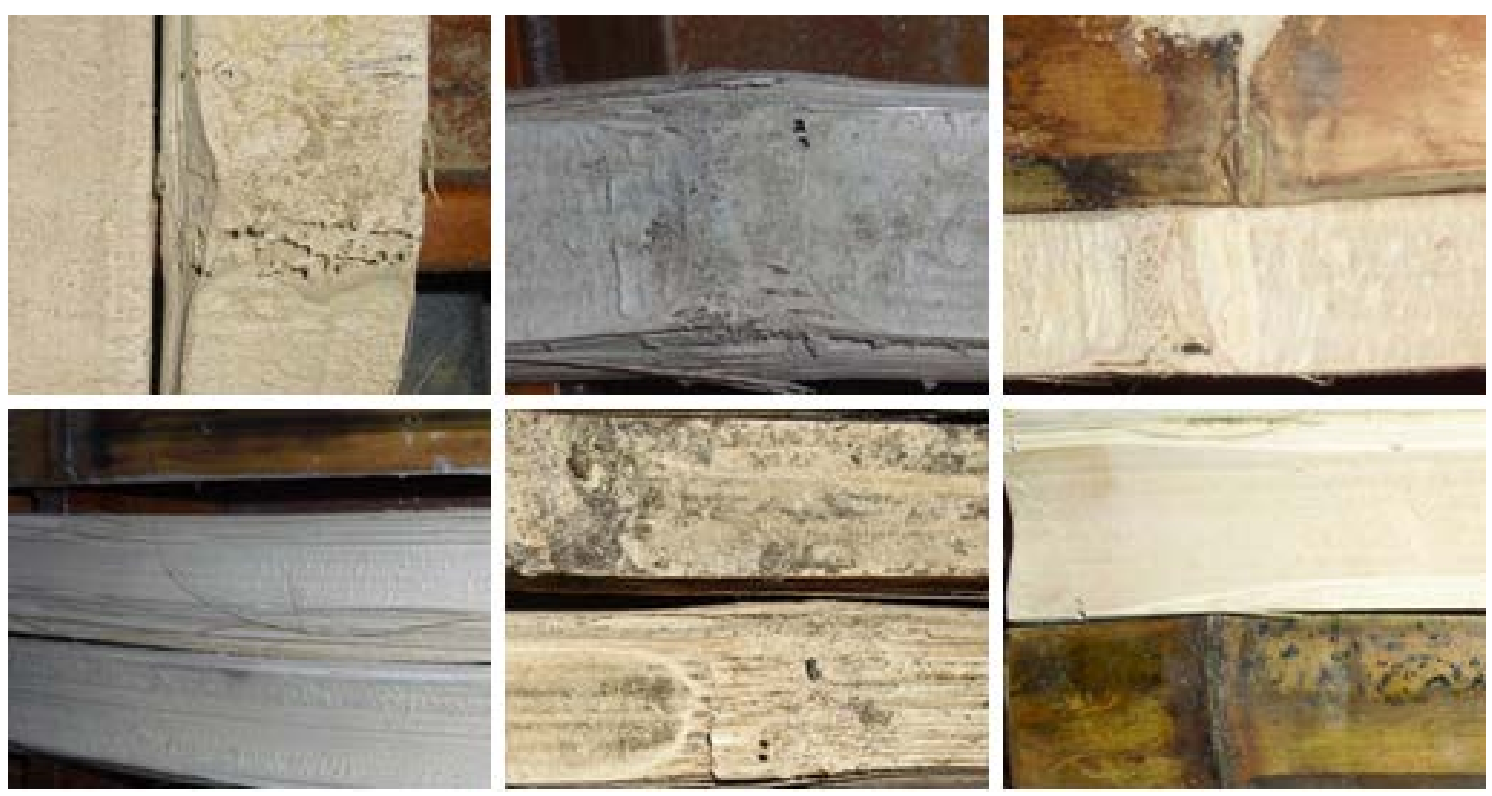

FIGURA 5. Ataque de Dinoderus minutus a especímenes de Bambusa vulgaris vittata sometidos a tratamientos por inmersión 

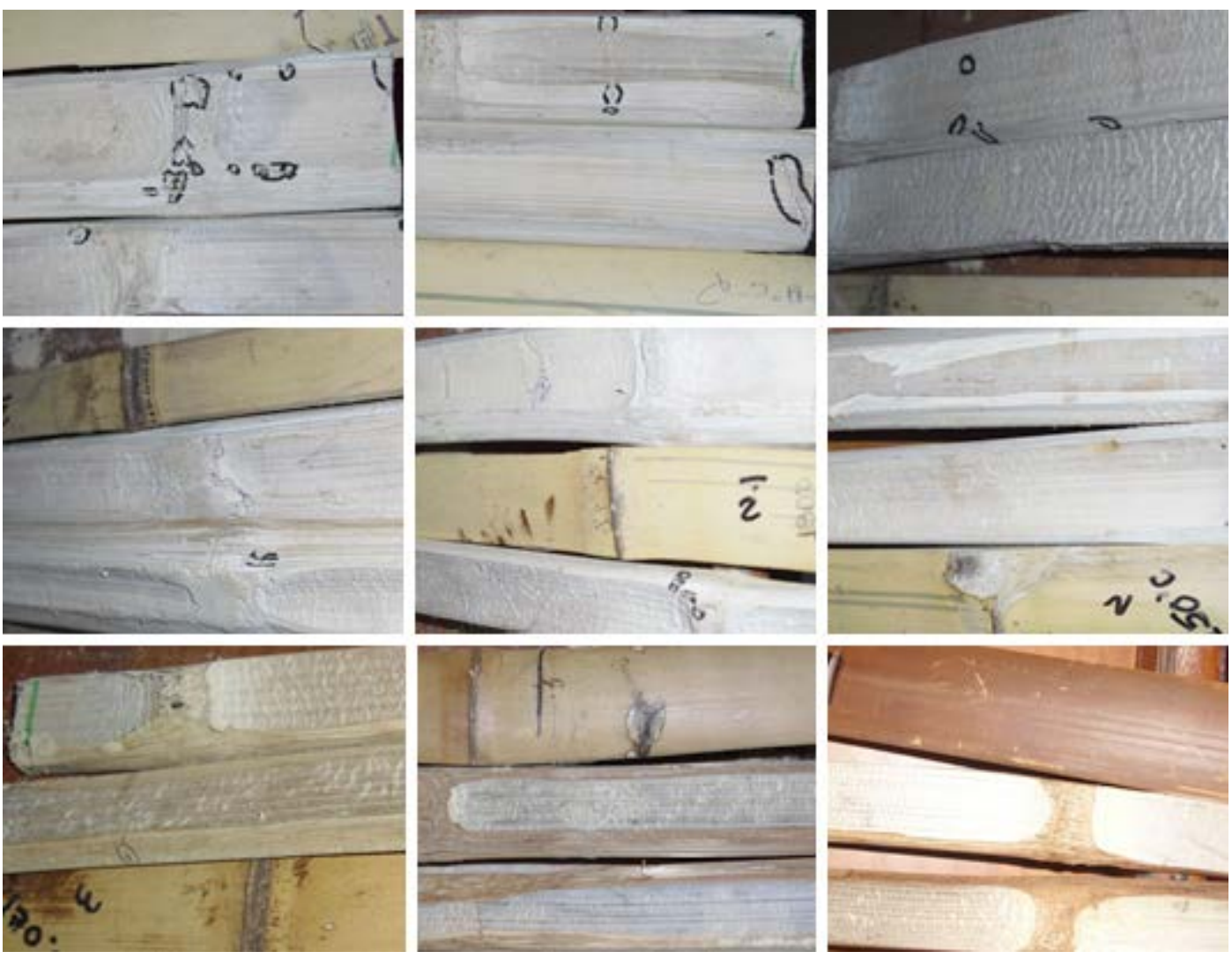

Figura 6. Ataque de Dinoderus minutus a especímenes de Bambusa vulgaris vittata sometidos a tratamientos por temperatura.

En la figura 7 se puede observar que todos los tratamientos, en comparación con el control, mostraron eficacia, reduciendo significativamente el ataque de los insectos, excepto los tratamientos térmicos de $50^{\circ} \mathrm{C}, 90^{\circ} \mathrm{C}$ y $130{ }^{\circ} \mathrm{C}$, en que los especímenes fueron severamente atacados.

El tratamiento térmico mostró un resultado más uniforme con la temperatura de $150^{\circ} \mathrm{C}$. En los resultados de las temperaturas más bajas se aprecia que, aunque el ataque puede considerarse bajo, hubo grandes oscilaciones. Esto pudiera deberse al lugar de obtención del espécimen con respecto al culmo completo, por ejemplo, al ser extraído de la parte basal de bambú donde la concentración de almidón es considerablemente superior.

\section{DISCUSIÓN}

Los resultados mostraron que todos los tratamientos fueron efectivos y redujeron considerablemente el ataque de los insectos, en comparación con el control (Tabla 1).
Por otro lado, y comparando los tratamientos preservadores, se observó que, similarmente a lo hallado por Kaur et al. (2014) en el tratamiento contra el ataque de la especie Dendrocalamus strictus, el extracto de Azadirachta indica no fue totalmente eficaz en comparación con otros tratamientos, a pesar de la reducción de su susceptibilidad. Por su parte, los otros tratamientos naturales presentaron buenos índices de preservación al exhibir menor cantidad de ataques (Fig. 7). Como se esperaba, el tratamiento basado en pentox fue bastante efectivo, a pesar de sus posibles daños ambientales. Además, no fue posible comparar con datos de la literatura por no existir trabajos similares para preservar los bambúes.

En cuanto al tratamiento térmico, se concluye que temperaturas de hasta $130^{\circ} \mathrm{C}$ no son eficaces para prevenir el ataque de Dinoderus minutus. En el intervalo de temperatura $150^{\circ} \mathrm{C}-170^{\circ} \mathrm{C}$, se concluye que la eficacia 


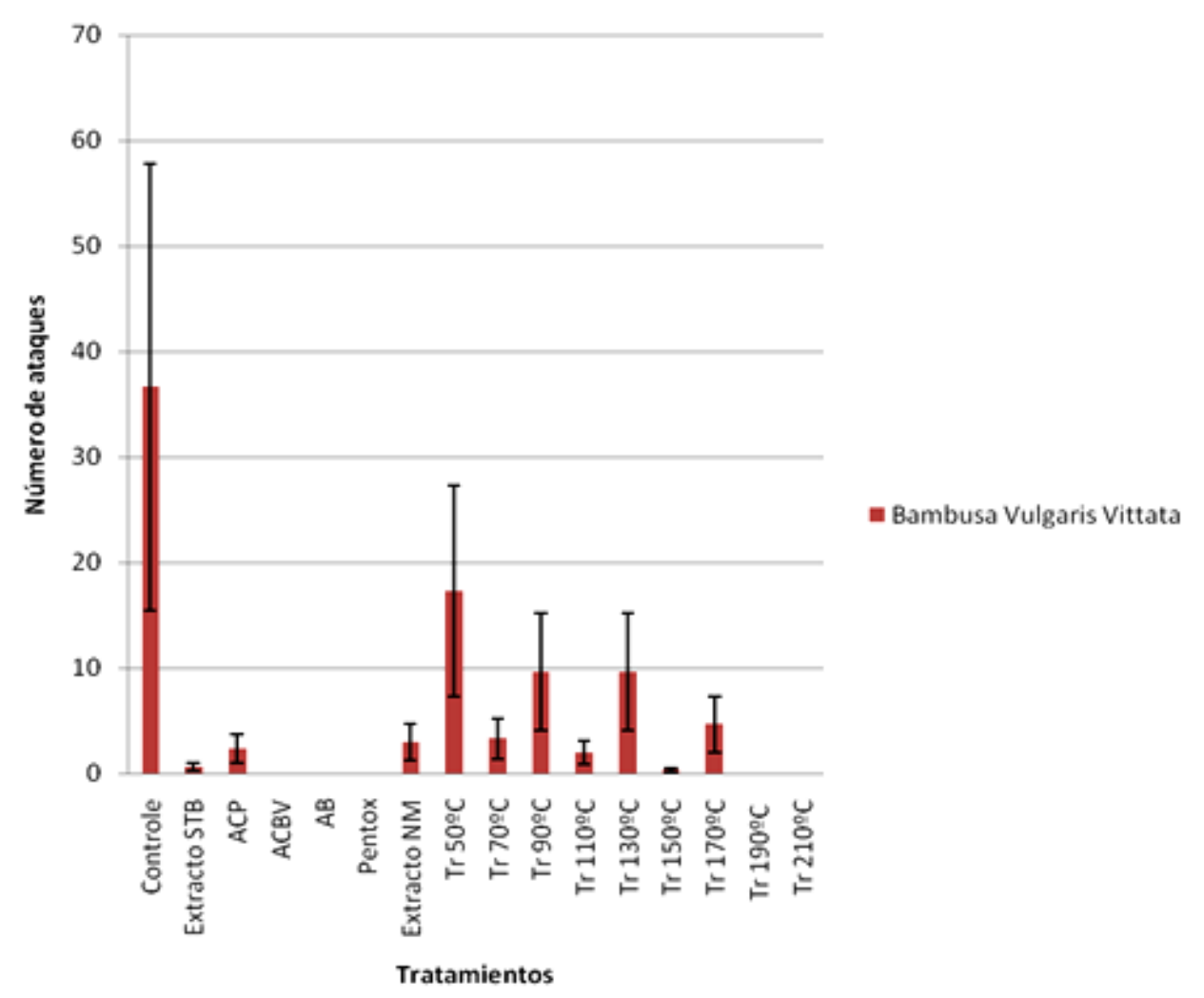

FIGURA 7. Número de ataques de Dinoderus minutus a Bambusa vulgaris vittata en relación con el tratamiento.

aumenta, observando pocos ataques de manera uniforme. Pero los mejores resultados obtenidos en relación con los ataques de insectos fueron a temperaturas de $190{ }^{\circ} \mathrm{C}$ y 210 ${ }^{\circ} \mathrm{C}$ (Tabla 1). En concordancia, en temperaturas mayores a $160{ }^{\circ} \mathrm{C}$, Zhang, Yu y Yu (2013) han verificado, para el tratamiento térmico de la especie Phyllostachys pubescens, que los contenidos de a-celulosa y holocelulosa se redujeron significantemente con ese incremento de temperatura. Los especímenes sometidos a tratamiento térmico con temperaturas desde $160{ }^{\circ} \mathrm{C}$ presentaron grietas en la región interior (Tuncer y Doğu, 2018); a pesar de esta situación, debida a la pérdida de peso por las altas temperaturas, se han obtenido mayores niveles de calidad y durabilidad del material (Zhang et al., 2013).

La variación en los resultados de los ataques puede ser justificada en función de posibles errores en el recuento. Es decir, en el recuento se puede confundir la entrada y la salida de los insectos, considerando uno como si fueran dos ataques. Siendo así, es necesario adoptar procedimientos más exactos para el recuento. En la mayor parte de los ejercicios, los Dinoderus minutus atacaron la región de los nodos (Figs. 5 y 6), lo que se esperaba por la alta concentración de almidón. También con respecto al almidón, la ubicación en el vástago interfiere con los resultados más próximos a la región basal, con menor densidad, la cual es más susceptible a ataques. Las partes del interior del bambú también concentran una mayor cantidad de almidón de acuerdo con la literatura y, por tanto, se observa que los ataques están concentrados en esa región interior.

\section{CONCLUSIONES}

Los tratamientos fueron eficaces en este estudio en comparación con la muestra control, probando que los productos naturales más amigables como los extractos (neem y cinamomo) y los ácidos piroleñosos (bambú y 
eucalipto) son buenas opciones para la preservación del bambú estudiado, el cual posee una de las mayores cantidades de almidón de todas las especies de bambú.

Sin embargo, deberán realizarse nuevas pruebas con diferentes tiempos de inmersión, la concentración de nuevos productos preservadores, otras especies de bambú y otros métodos para lograr una mejor impregnación, así como una mejor eficiencia de los tratamientos.

Este estudio dará pie a nuevas investigaciones y metodologías a seguir para el trabajo futuro, en busca de mejorar la eficacia de los preservadores, que sean menos tóxicos para el ambiente y los seres humanos, frente al ataque de los gorgojos en los bambúes.

\section{REFERENCIAS}

Arruda, L. M., del Menezzi, C. H. S., Teixeira, D. E., \& de Araújo P. C. (2011). Lignocellulosic composites from Brazilian giant bamboo (Guadua magna). Part 1: properties of resin bonded particleboards. Maderas Ciencia y Tecnología, 13(1), 4958. doi: 10.4067/S0718-221X2011000100005

Azzini, A. \& Beraldo, A. L. (2000). Determinação de fibras celulósicas e amido em cavacos laminados de três espécies de bambu gigante. Scientia Forestalis, 57, 45-51.

Barboza, A. S. R., Barbirato, J. C. C., \& Silva, M. M. C. P. 2(008). Avaliação do uso de bambu como material alternativo para a execução de habitação de interesse social. Ambiente Construído, 8(1), 115-129.

Brito, J. O., Garcia, J. N., Bortoletto Junior, G., Pessoa, A. M. C, \& Silva, P. H. M. (2006). Densidade básica e retratibilidade da madeira de Eucalyptus grandis, submetida a diferentes temperaturas de termorretificação. Cerne, 12(2),182-188.

Colla, W. A., Beraldo, A. L., \& Brito, J. O. (2011). Effects of thermal treatment on the physicochemical 361 characteristics of giant bamboo. Cerne, 17(3), 361-367.

Diver, S. (2001). Bamboo: a multipurpose agroforestry crop. Butte, Estados Unidos: ATTRA/NCAT.

European Committee for Standardization (2000). European Standard EN 310 Placas de derivados de madeira: determinação do módulo de elasticidade em flexão e da resistência a flexão., Lisbon, Portugal: European Committee for Standardization.

Escamilla, E. Z., Habert, G., \& Wohlmuth, E. (2016). When $\mathrm{CO}_{2}$ counts: sustainability assessment of industrialized bamboo as an alternative for social housing programs in the Philippines. Building and Environment, 103, 44-53. Doi: 10.1016/j.buildenv.2016.04.003
Espelho, J. C. C. \& Beraldo, A. L. (2008). Avaliação físicomecânica de colmos de bambu tratados. Agriambi, 12(6), 645-652.

Galvão, A. P. M. (1967). Tratamento preservativo do bamboo pelo processo do banho frio. Anais da Escola Superior de Agricultura Luiz de Queiroz, 24, 19-33.

Kaur, P., Pant, K. K., Satya, S., \& Naik, S. N. (2014). Comparison of decay resistance of bamboo treated with plant extracts and oil cakes. International Journal of Emerging Technology and Advanced Engineering, 4(1), 582-585.

Kurhekar, S. P., Moola, L. R., Shinde, N. V., \& Kolhe, P. R. (2015a). Development and testing of pressurized bamboo treatment unit. International Journal of Tropical Agriculture, 33(2), 1703-1705.

Kurhekar, S. P., Sanap, P. B., \& Kolhe, P. R. (2015b). Studies on mechanical properties of treated bamboo. International Journal of Tropical Agriculture, 33(2), 1687-1690.

Liese, W. (1994). Biological aspects of bamboo and rattan for quality improvement by polymer impregnation. Folia Forestalia Polonica, B(25), 43-56.

Liese, W. 1980. Preservation of bamboos. In: Lessard G., A. Chouinard (eds). Bamboo Research in Asia. IDRC. Ottawa, Canada. p:165-172.

Liese, W. \& Tang, T. K. H. (2015). Preservation and drying of bamboo. En W. Liese, \& M. Köhl. Bamboo: the plant and its uses (pp. 257-297). Heidelberg, Alemania: Springer.

Meng, F., Yu, Y., Zhang, Y., Yu, W., \& Gao, J. (2016). Surface chemical composition analysis of heat-treated bamboo. Applied Surface Science, 371, 383-390. doi: 10.1016/j.apsusc.2016.03.015

Nunes, A. R. S. (2005). Construindo com a natureza bambu: uma alternativa de ecodesenvolvimento. Tesis de Maestría, Universidade Federal do Sergipe (UFS), São Cristóvão, Brasil.

Obiri, B. D. \& Oteng-Amoako A. A. (2007). Towards a sustainable development of the bamboo industry in Ghana. Ghana Journal of Forestry, 21-22(1), 14-27.

Ordóñez-Candelaria, V. R. (1999). Perspectivas del bambú para la construcción en México. Madera y Bosques, 5(1), 3-12. doi: $10.21829 /$ myb.1999.511350

Ordóñez-Candelaria, V. R. \& Salomón-Quintana, I. (2009). Consideraciones geométricas en la determinación de las propiedades en flexión estática de bambú. Madera y Bosques, 15(1), 91-100. doi: 10.21829/myb.2009.1511199 
Pereira, M. A. R. \&Beraldo, A. L. (2007). Bambu de corpo e alma. Bauru, Brasil: UNESP.

Schäfer, K., Goergen, G., \& Borgemeister, C. (2000). An illustrated identification key to four different species of adult Dinoderus (Coleoptera: Bostrichidae), commonly attacking dried cassava chips in West Africa. Journal of Stored Products Research, 36(2000), 245-252. doi: 10.1016/S0022474X(99)00046-6

Singh, P. \& Bhandari, R. S. (1988). Insect pests of bamboo and their control. Indian Forester, 114(10), 670-683.

Tiburtino, R. F., Paes, J. B., Beraldo, A. L., Arantes, M. D. C., \& Brocco V. F. (2015). Tratamento preservativo de duas espécies de bambu por imersão prolongada e Boucherie modificado. Floresta e Ambiente, 22(1), 124-133. doi: 10.1590/2179-8087.032113

Tuncer, F. D. \& Doğu, A. D. (2018). Effects of heat treatment on some macroscopic and physical properties of Scots pine sapwood and heartwood. Forestist, 68(2), 93-100. doi: 10.26650 / forestist.2018.343295

Vasconcellos, R. M. (2004). Cartilha de fabricação de móveis. Maceió, Brasil: Instituto do Bambu.

Zaragoza-Hernández, I., Borja de la Rosa, A., Zamudio-Sánchez, F. J., Ordóñez-Candelaria, V. R., \& Bárcenas-Pazos, G. M.
(2014). Anatomía del culmo de bambú (Guadua aculeata Rupr.) de la región nororiental del estado de Puebla, México. Madera y Bosques, 20(3), 87-96. doi: 10.21829/myb.2014.203154

Zhang, Y. M., Yu, Y. L., \& Yu, W. J. (2013). Effect of thermal treatment on the physical and mechanical properties of Phyllostachys pubescens bamboo. European Journal of Wood Products, 71(1), 61-67. doi: 10.1007/s00107-012-0643-6

Manuscrito recibido el 27 de enero de 2017

Aceptado el 11 de octubre de 2019

Publicado el 19 de agosto de 2019

Este documento se debe citar como:

Cortez-Barbosa, J., Morales, E. A. M., Higuti, K. A., Alves, A. C. L., De Araujo, V. A., Gava, M., \& Garcia, J. N. (2019). Tratamientos de preservación de Bambusa vulgaris vittata contra el ataque de Dinoderus minutus. Madera y Bosques, 25(2), e252550. doi: 10.21829/myb.2019.252550

Madera y Bosques por Instituto de Ecología, A.C. se distribuye bajo una Licencia Creative Commons Atribución-NoComercialCompartirlgual 4.0 Internacional. 